2

3

4

5

6

7 Wei E. Huang ${ }^{1}$, Shuang-Jiang Liu ${ }^{2}$, Xiao-Lei Wu ${ }^{3}$, Yu-Zhong Zhang ${ }^{4}$ and Fengping Wang ${ }^{5}$

8

9 1. Department of Engineering Science, University of Oxford, Parks Road, Oxford, OX1

10

11

12

13

14

15

16

17

18

19

\section{Environmental Microbiology in China}

by 3PJ, United Kingdom.

2. State Key Laboratory of Microbial Resources, Institute of Microbiology, Chinese Academy of Sciences, Beijing, China

3. College of Engineering, Peking University, Beijing 100871, China

4. State Key Laboratory of Microbial Technology, Shandong University, Qingdao 266237, China

5. State Key Laboratory of Microbial Metabolisms, School of Sciences and Biotechnology; School of Oceanography, Shanghai JiaoTong University, Minhang, Shanghai 200240, China 
1Since the new millennia, China has made up about a quarter of global Research and 2Development funding on science and technology. The funding has significantly promoted 3China's advances in basic and applied microbiology and biotechnology. China's rapid 4economy growth has also stimulated environmental microbiology research in various areas 5 such as agriculture, aquaculture, deep sea ecosystems, green bioenergies, and bioremediation. 6Environmental microbiology research has a long history in China and it becomes a hot topic 7over the past decades for three reasons. 1) China's economic boost in the past years left a 8legacy of environmental pollution, posing a challenge to environmental microbiology. 2) 9Increasing concerns of environmental quality have been raised from the public and 10government, and the applications of environmental microorganisms to the protection and 11improvement of environments, including water, air and soil, have been successfully 12demonstrated. 3) Both government and research funding bodies strongly supported basic 13research and application research on environmental microbiology. For example, National 14Natural Science Foundation of China (NSFC) has funded 613 projects with 347.8 million 15RMB for environmental microbiology research during the period of 2010-2019, and 141 16universities and 69 research institutions were involved. Environmental Microbiology has 17firmly become an important research area in China, and a special Environmental 18Microbiology Committee under Chinese Society for Microbiology attracts thousands of 19members.

20As one of the top journals in the field of environmental microbiology, Environmental 21Microbiology (EMI) and Environmental Microbiology Reports (EMIR) created this special 22issue, collecting and exampling Chinese contributions to environmental microbiology.

23Here in this special issue of 'Advances in Environmental Microbiology in China', Chinese 24microbiologists, together with their international collaborators, explored broad topics of 25environmental microbiology, from microbial ecology, biogeography, microbial physiology, 26evolution to gene regulations and mining novel enzymes from uncultivable microbial 27communities. They asked some fundamental questions of environmental microbiology: "who 28are there? what are they doing? how they do it? and can we make use of them?". They 29investigated microorganisms in different habitats, including the interaction of microbes and 30viruses in rhizosphere and ocean, $\mathrm{CO}_{2}$ fixation in paddy soils, salinity control the structure of 
1microbial community in wetland, biodegradation of polycyclic aromatic hydrocarbon 2biodegradation in river ecosystem, bacteria impact on microcystis blooms in lake, microbial 3adaption mechanisms in sediment under seafloor. Studies on biogeochemistry, element 4cycles, microbial physiology, the interactions between microbes and virus and plants were 5 also documented in this issue. In terms of environmental molecular biology, gene regulations, 6 functional genes and enzyme characterisation, and genetic evolution were elegantly studied. 7To exploit environmental microorganisms, a study has used uncultured microbial 8 communities as genetic resource to mine functional enzymes.

9The contributors of this special issue of Environmental Microbiology (EMI) and 10Environmental Microbiology Reports (EMIR) are microbiologists from the Chinese 11Academy of Sciences, research institutes and universities.

12

\section{Acknowledgement}

14We thank Dr Quan-Sheng Du in Chinese NSFC for helpful advice.

15

16

17 